\title{
A Mean-Variance Portfolio Selection Model with Interval-Valued Possibility Measures
}

\author{
Yunyun Sui $\mathbb{D}^{1}{ }^{1}$ Jiangshan $\mathrm{Hu},{ }^{1}$ and Fang $\mathrm{Ma}^{2}$ \\ ${ }^{1}$ School of Mathematics and Information Science, Weifang University, Weifang 261061, China \\ ${ }^{2}$ School of Science, Shenyang University of Technology, Shenyang 110023, China \\ Correspondence should be addressed to Yunyun Sui; suiyunyun1231@163.com
}

Received 27 January 2020; Revised 14 July 2020; Accepted 1 August 2020; Published 29 August 2020

Academic Editor: Anna M. Gil-Lafuente

Copyright (c) 2020 Yunyun Sui et al. This is an open access article distributed under the Creative Commons Attribution License, which permits unrestricted use, distribution, and reproduction in any medium, provided the original work is properly cited.

In recent years, fuzzy set theory and possibility theory have been widely used to deal with an uncertain decision environment characterized by vagueness and ambiguity in the financial market. Considering that the expected return rate of investors may not be a fixed real number but can be an interval number, this paper establishes an interval-valued possibilistic mean-variance portfolio selection model. In this model, the return rate of assets is regarded as a fuzzy number, and the expected return rate of assets is measured by the interval-valued possibilistic mean of fuzzy numbers. Therefore, the possibilistic portfolio selection model is transformed into an interval-valued optimization model. The optimal solution of the model is obtained by using the order relations of interval numbers. Finally, a numerical example is given. Through the numerical example, it is shown that, when compared with the traditional possibilistic model, the proposed model has more constraints and can better reflect investor psychology. It is an extension of the traditional possibilistic model and offers greater flexibility in reflecting investor expectations.

\section{Introduction}

Markowitz's mean-variance model, proposed in 1952, brought portfolio theory into the quantitative era [1]. Since then, many portfolio selection models (e.g., [2-5]) have been established, and there are now several different ways to characterize the risk of assets such as by using the semivariance risk measure [6], the mean absolute deviations risk measure [7], the semiabsolute deviation risk measure [8], and the value-at-risk measure (abbreviated as VaR) [9-11].

In the mean-variance model, the expected rate of return of a portfolio is regarded as a random variable, and the mean and variance of the random variable are taken as the expected return and risk of the portfolio, respectively. However, there are many nonprobabilistic factors, such as social, economic, political, and psychological factors, in real-world portfolio decision-making. In addition, investors may also face imprecise information and therefore need to deal with imprecise, fuzzy, and ambiguous information [12]. In this case, using probability theory to solve problems will yield counterintuitive results $[13,14]$. Consequently, researchers find that asset returns can be estimated by fuzzy set theory [15].

In recent years, many scholars have tried to employ fuzzy variables to manage portfolio selection problems and built many fuzzy portfolio models [16-21]. In 2013, Tsaur [22] constructed a fuzzy portfolio model with the parameters of fuzzy-input return rates and fuzzy-output proportions. Zhou et al. [23] proposed the concept of fuzzy semientropy. They used semientropy to quantify the downside risk and set up two mean-semientropy portfolio selection models. To obtain the optimal solution, they used the genetic algorithm. Based on credibility theory, a class of mean-variance adjusting models with transaction costs was proposed in [24].

Possibility theory is an important theory of fuzzy sets that was first proposed by Zadeh [25] and developed by Dubois and Prade [26] (see $[27,28]$ for more detail). In possibility theory, the relationship between fuzzy variables and possibility distributions is the same as that between random variables and probability distributions in 
probability theory. Tanaka et al. [29] first proposed the possibilistic portfolio selection model. Carlsson and Fullér [30] then introduced the notions of lower and upper possibilistic mean values as well as the notations for crisp possibilistic mean value and variance of continuous possibility distributions. Based on that, Carlsson et al. [31] assumed that the returns of securities are trapezoidal fuzzy variables and found the optimal portfolio model with the highest utility score. Zhang and Nie [32] extended the possibilistic mean and variance concepts proposed by Carlsson and Fullér [30] and presented the notions of upper and lower possibilistic variances and covariances of fuzzy numbers. Fullér and Majlender [33] introduced the notation of weighted interval-valued possibilistic mean value of fuzzy numbers. Zhang and Wang [34] proposed a possibilistic mean-variance model. Zhang [35] proposed lower and upper possibilistic meanstandard deviation model variance models. Zhang et al. [36] proposed two kinds of portfolio selection models based on the upper and lower possibilistic means and variances introduced by Carlsson and Fullér [30] and Zhang and Nie [32].

In order to better integrate an uncertain decision environment characterized by vagueness and ambiguity, Zhang et al. [37] proposed a possibilistic mean-variance portfolio selection model based on the definitions of the possibilistic return and possibilistic risk. In this model, the return rate of assets is regarded as a fuzzy variable with LRtype possibility distribution, and the possibilistic mean of the fuzzy variable is taken as the expected return rate of the asset. However, the possibilistic portfolio model mentioned above only considers that investors hope that the return (rate) of their portfolio will reach an expected value. In real financial markets, investors hope that the expected return (rate) of their portfolio will not be an exact value, but a range of values. In other words, investors want their return (rate) on assets to be within a certain range. Thus, an interval-valued possibilistic mean-variance portfolio selection model is proposed. In this model, we will use the interval-valued possibilistic mean of fuzzy numbers proposed by [30] to measure the expected return of assets, so the portfolio selection model is transformed into an interval-valued programming model. See Section 3 for a detailed description of the model.

In recent years, many researchers have studied interval-valued programming and obtained fruitful results [38-41]. Tong [42] reduced interval number linear programming into two types of classical linear programming by introducing a maximum value range and obtained an interval number optimal solution. Lai et al. [43] defined the noninferior solutions to a class of linear programming problems with interval coefficients in both the objective functions and constraints based on two order relations between intervals. The order relation of interval numbers plays an important role in solving interval programming. Ishibuchi and Tanaka $[44,45]$ gave two order relations of interval numbers. To obtain a solution for the intervalvalued programming model, we use the order relation of interval numbers to transform the interval-valued programming model into a quadratic programming model. In the model, the interval number is used to describe the expected return rate of a portfolio, and the lower limit of the expected return rate of investors is not limited to a fixed real number but can be an interval number. This can better describe the psychology of investors and give investors a more flexible choice and help them make choices with greater flexibility.

The rest of this paper is organized as follows. In Section 2 , we present some basic concepts regarding possibility theory and notions of the possibilistic mean and variance of a fuzzy number. At the same time, in this section, we recall the notion of interval numbers and their order relations. In Section 3, we propose an interval-valued possibilistic portfolio selection model. We suppose that the return rates of the assets are trapezoidal fuzzy numbers and then use the interval-valued possibilistic mean to measure the expected return rate of assets. To obtain the solution for the intervalvalued programming model, the order relation of interval numbers is used to transform the model into a quadratic programming model. Section 4 provides a numerical example to illustrate the proposed approach. Section 5 discusses the proposed model and compares it with the traditional possibilistic model. Through this comparison, the proposed model is found to have more constraints and is able to better reflect investor psychology. It is an extension of the traditional possibilistic model and has greater flexibility in reflecting investor expectations. Section 6 provides the conclusion.

\section{Preliminaries}

In this paper, concepts and operations related to fuzzy sets and interval numbers will be used. This section will briefly review the relevant concepts.

Definition 1. A fuzzy number $A$ is a fuzzy set of the real line $\mathbb{R}$ with a normal, fuzzy convex and continuous membership function of bounded support. The family of fuzzy numbers will be denoted by $\mathscr{F}$.

Definition 2. The $\lambda$-level set of fuzzy number $A$ is denoted by

$$
A^{\lambda}= \begin{cases}\left\{x \in \mathbb{R} \mid \mu_{A}(x) \geq \lambda\right\}, & \lambda>0, \\ \operatorname{cl}\left\{x \in \mathbb{R} \mid \mu_{A}(x)>0\right\}, & \lambda=0,\end{cases}
$$

where $\mathrm{cl}$ represents the closure of the support of $A$. The $\lambda$-level set of $A$ is expressed as $A^{\lambda}=[a(\lambda), \mathrm{b}(\lambda)](\lambda>0)$ (See Dubois and Prade [46]).

Definition 3. A fuzzy number $A$ is called the trapezoidal fuzzy number, denoted by $A=(a, b, \alpha, \beta)$ if its membership has the following form: 


$$
\mu(x)= \begin{cases}1-\frac{a-x}{\alpha}, & a-\alpha \leq x \leq a, \\ 1, & a \leq x \leq b, \\ 1-\frac{x-b}{\beta}, & b \leq x \leq b+\beta \\ 0, & \text { otherwise }\end{cases}
$$

where the interval $[a, b]$, the real number $\alpha$, and the real number $\beta$ are called the tolerance interval, the left width, and the right width, respectively.

Remark 1. If $a=b$ in (2), then $A$ is called a triangular fuzzy number.

Remark 2. If $A=(a, b, \alpha, \beta)$, then its $\lambda$-level set can easily be shown like this: $A^{\lambda}=[a-(1-\lambda) \alpha, b+(1-\lambda) \beta]$, where $\lambda \in[0,1]$.

The definitions of the possibilistic mean, variance, covariance, and interval-valued possibilistic mean of a fuzzy number were given by Carlsson and Fullér [30] as follows.

Definition 4. The upper possibilistic mean value of $A$ with $\lambda$-level set $A^{\lambda}=[a(\lambda), \mathrm{b}(\lambda)]$ is defined as

$$
M^{*}(A)=\frac{\int_{0}^{1} \operatorname{Pos}[A \geq b(\alpha)] b(\alpha) \mathrm{d} \alpha}{\int_{0}^{1} \operatorname{Pos}[A \geq b(\alpha)] \mathrm{d} \alpha}=2 \int_{0}^{1} \alpha b(\alpha) \mathrm{d} \alpha
$$

where Pos denotes the possibility measure.

Definition 5. The lower possibilistic mean value of $A$ is defined as

$$
M_{*}(A)=\frac{\int_{0}^{1} \operatorname{Pos}[A \leq a(\alpha)] a(\alpha) \mathrm{d} \alpha}{\int_{0}^{1} \operatorname{Pos}[A \leq a(\alpha)] \mathrm{d} \alpha}=2 \int_{0}^{1} \alpha a(\alpha) \mathrm{d} \alpha .
$$

Definition 6. The interval-valued possibilistic mean of $A$ is defined as

$$
E(A)=\left[M_{*}(A), M^{*}(A)\right]
$$

Definition 7. The crisp possibilistic mean value of $A$ is defined as

$$
M(A)=\frac{M_{*}(A)+M^{*}(A)}{2}=\int_{0}^{1} \alpha(a(\alpha)+b(\alpha)) \mathrm{d} \alpha .
$$

Definition 8. The possibilistic variance of $A$ is defined as

$$
\begin{aligned}
\operatorname{Var}(A)= & \int_{0}^{1} \operatorname{Pos}[A \leq a(\alpha)]\left(\left[\frac{a(\alpha)+b(\alpha)}{2}-a(\alpha)\right]^{2}\right) \mathrm{d} \alpha \\
& +\int_{0}^{1} \operatorname{Pos}[A \geq b(\alpha)]\left(\left[\frac{a(\alpha)+b(\alpha)}{2}-b(\alpha)\right]^{2}\right) \mathrm{d} \alpha \\
= & \int_{0}^{1} \alpha\left(\left[\frac{a(\alpha)+b(\alpha)}{2}-a(\alpha)\right]^{2}\right) \mathrm{d} \alpha \\
& +\int_{0}^{1} \alpha\left(\left[\frac{a(\alpha)+b(\alpha)}{2}-b(\alpha)\right]^{2}\right) \mathrm{d} \alpha \\
= & \frac{1}{2} \int_{0}^{1} \alpha[b(\alpha)-a(\alpha)]^{2} \mathrm{~d} \alpha .
\end{aligned}
$$

Definition 9. The possibilistic covariance between fuzzy numbers $A$ and $B$ is defined as

$$
\operatorname{Cov}(A, B)=\frac{1}{2} \int_{0}^{1} \alpha\left(b_{1}(\alpha)-a_{1}(\alpha)\right)\left(b_{2}(\alpha)-a_{2}(\alpha)\right) \mathrm{d} \alpha .
$$

Lemma 1. Let $\alpha, \beta \in R$, and let $A$ and $B$ be fuzzy numbers, and then

$$
\operatorname{Var}(\alpha A+\beta B)=\alpha^{2} \operatorname{Var}(A)+\beta^{2} \operatorname{Var}(B)+2|\alpha \beta| \operatorname{Cov}(A, B) .
$$

Example 1. Let $A=(a, b, \alpha, \beta)$ be a trapezoidal fuzzy number, and then its $\lambda$-level set is $A^{\lambda}=[a-(1-\lambda) \alpha, b+(1-\lambda) \beta]$.

So, the lower possibilistic mean value of $A$ is

$$
M_{*}(A)=2 \int_{0}^{1} \lambda a(\lambda) \mathrm{d} \lambda=2 \int_{0}^{1} \lambda[a-(1-\lambda) \alpha] \mathrm{d} \lambda=a-\frac{\alpha}{3},
$$

and the upper possibilistic mean value of $A$ is

$$
M^{*}(A)=2 \int_{0}^{1} \lambda b(\lambda) \mathrm{d} \lambda=2 \int_{0}^{1} \lambda[b+(1-\lambda) \beta] \mathrm{d} \lambda=b+\frac{\beta}{3} .
$$

So, the interval-valued possibilistic mean of $A$ is

$$
\begin{aligned}
\mathrm{E}(A) & =\left[a-\frac{\alpha}{3}, b+\frac{\beta}{3}\right], \\
\operatorname{Var}(A) & =\frac{1}{2} \int_{0}^{1} \lambda[b(\lambda)-a(\lambda)]^{2} \mathrm{~d} \lambda \\
& =\frac{1}{2} \int_{0}^{1} \lambda[b+(1-\lambda) \beta-a+(1-\lambda) \alpha]^{2} \mathrm{~d} \lambda \\
& =\frac{1}{24}\left[6(b-a)^{2}+(\beta+\alpha)^{2}+4(b-a)(\beta+\alpha)\right] .
\end{aligned}
$$


Example 2. Given two trapezoidal fuzzy numbers $A=\left(a_{1}, b_{1}, \alpha_{1}, \beta_{1}\right)$ and $B=\left(a_{2}, b_{2}, \alpha_{2}, \beta_{2}\right)$,

$$
\begin{aligned}
\operatorname{Cov}(A, B) & =\frac{1}{2} \int_{0}^{1} \lambda\left(b_{1}(\lambda)-a_{1}(\lambda)\right)\left(b_{2}(\lambda)-a_{2}(\lambda)\right) \mathrm{d} \lambda \\
& =\frac{1}{2} \int_{0}^{1} \lambda\left[b_{1}+(1-\lambda) \beta_{1}-a_{1}+(1-\lambda) \alpha_{1}\right]\left[b_{2}+(1-\lambda) \beta_{2}-a_{2}+(1-\lambda) \alpha_{2}\right] \mathrm{d} \lambda \\
& =\frac{1}{24}\left[6\left(b_{1}-a_{1}\right)\left(b_{2}-a_{2}\right)+\left(\beta_{1}+\alpha_{1}\right)\left(\beta_{2}+\alpha_{2}\right)+2\left[\left(b_{1}-a_{1}\right)\left(\beta_{2}+\alpha_{2}\right)+\left(b_{2}-a_{2}\right)\left(\beta_{1}+\alpha_{1}\right)\right]\right] .
\end{aligned}
$$

Remark 3. For trapezoidal fuzzy number $A=(a, b, \alpha, \beta)$, if $\alpha=\beta=0$, then $A=[a, b]$.

Thus, the interval number is a special fuzzy number, which Hansen [47] and Alefeld and Herzberger [48] discussed in detail. The operations related to interval numbers are as follows.

Definition 10. Let $a=\left[a_{L}, a_{U}\right], b=\left[b_{L}, b_{U}\right]$ be two intervals, and then

(i) $a \pm b=\left[a_{L} \pm b_{L}, a_{U} \pm b_{U}\right]$

(ii) $k a= \begin{cases}{\left[k a_{L}, k a_{U}\right],} & \text { for } k \geq 0, \\ {\left[k a_{U}, k a_{L}\right],} & \text { for } k<0 .\end{cases}$

The order relation of interval numbers plays an important role in solving interval programming, so Ishibuchi and Tanaka $[44,45]$ gave the following two order relations of interval numbers.

Definition 11. Let $a=\left[a_{L}, a_{U}\right]$ and $b=\left[b_{L}, b_{U}\right]$ be two interval numbers. We define the two order relations $\preccurlyeq_{1}$ and $\preccurlyeq_{2}$ between $a$ and $b$ as

(i) $a \preccurlyeq_{1} b$, if and only if $a_{L} \leq b_{L}$ and $\frac{a_{L}+a_{U}}{2} \leq \frac{b_{L}+b_{U}}{2}$,

$$
a \prec_{1} b, \quad \text { if and only if } a \preccurlyeq_{1} b \text { and } a \neq b \text {, }
$$

(ii) $a \leqslant_{2}$ b, if and only if $a_{U} \leq b_{U}$ and $\frac{a_{L}+a_{U}}{2} \leq \frac{b_{L}+b_{U}}{2}$,

$$
a \prec_{2} b, \quad \text { if and only if } a \preccurlyeq_{2} b \text { and } a \neq b \text {. }
$$

\section{Model Foundation}

In this section, we set up an interval-valued possibilistic meanvariance portfolio selection model. To reflect the investor's investment elasticity more flexibly, we use possibility theory to construct an interval-valued possibilistic portfolio model.

Suppose that there are $n$ risky assets and one risk-free asset available for investment. Let $r_{k}$ be the return rate of asset $k, k=1,2, \ldots, n$, which is a fuzzy number. Let $x_{k}$ represent the proportion invested in asset $k, k=1,2, \ldots, n$, and let $r_{f}$ be the return of the risk-free asset.

Thus, the portfolio's return $R$ can be written as

$$
R=\sum_{k=1}^{n} x_{k} r_{k}+r_{f}\left(1-\sum_{k=1}^{n} x_{k}\right) .
$$

Obviously, $R$ is a fuzzy number.

To set up a portfolio selection model, the following values need to be given.

First, the interval-valued possibilistic mean of the portfolio's return $R$ is given by

$$
\begin{aligned}
E(R) & =E\left(\sum_{k=1}^{n} x_{k} r_{k}+r_{f}\left(1-\sum_{k=1}^{n} x_{k}\right)\right) \\
& =\sum_{k=1}^{n} x_{k} E\left(r_{k}\right)+r_{f}\left(1-\sum_{k=1}^{n} x_{k}\right) \\
& =\sum_{k=1}^{n} x_{k}\left[M_{*}\left(r_{k}\right), M^{*}\left(r_{k}\right)\right]+r_{f}\left(1-\sum_{k=1}^{n} x_{k}\right) .
\end{aligned}
$$

Second, the possibilistic variance of $R$ is as follows:

$$
\begin{aligned}
\operatorname{Var}(R) & =\sum_{k=1}^{n} x_{k}^{2} \operatorname{Var}\left(r_{k}\right)+2 \sum_{i>j=1}^{n}\left|x_{i} x_{j}\right| \operatorname{Cov}\left(r_{i}, r_{j}\right) \\
& =\sum_{k=1}^{n} x_{k}^{2} \operatorname{Var}\left(r_{k}\right)+2 \sum_{i>j=1}^{n} x_{i} x_{j} \operatorname{Cov}\left(r_{i}, r_{j}\right)
\end{aligned}
$$

According to the mean-variance model, we establish the following portfolio model: 


$$
\left\{\begin{array}{l}
\min \quad \operatorname{Var}(R) \\
\text { s.t. } \quad E(R) \geq \bar{\mu} \\
\sum_{k=1}^{n} x_{k} \leq 1 \\
0 \leq d_{k} \leq x_{k} \leq g_{k}, k=1,2, \ldots, n
\end{array}\right.
$$

where $\bar{\mu}$ is a minimum threshold at which investors can tolerate the expected rate of return on their portfolio, set $\bar{\mu}=\left[\mu^{-}, \mu^{+}\right] . d_{k}$ and $g_{k}$ represent, respectively, the lower and the upper bounds on investment in asset $k, k=1,2, \ldots, n$.

Equations (19)-(21) can be written as

$$
\left\{\begin{array}{l}
\min \quad \operatorname{Var}(R)=\sum_{k=1}^{n} x_{k}^{2} \operatorname{Var}\left(r_{k}\right)+2 \sum_{i>j=1}^{n} x_{i} x_{j} \operatorname{Cov}\left(r_{i}, r_{j}\right) \\
\text { s.t. } \quad \sum_{k=1}^{n} x_{k}\left[M_{*}\left(r_{k}\right), M^{*}\left(r_{k}\right)\right]+r_{f}\left(1-\sum_{k=1}^{n} x_{k}\right) \geq \bar{\mu} \\
\sum_{k=1}^{n} x_{k} \leq 1 \\
0 \leq d_{k} \leq x_{k} \leq g_{k}, k=1,2, \ldots, n .
\end{array}\right.
$$

Let $r_{k}$, the return on asset $k$, be a trapezoidal fuzzy number $r_{k}=\left(a_{k}, b_{k}, \alpha_{k}, \beta_{k}\right)$. Then, we have

$$
\begin{aligned}
\operatorname{Var}(R)= & \sum_{k=1}^{n} x_{k}^{2} \operatorname{Var}\left(r_{k}\right)+2 \sum_{i>j=1}^{n} x_{i} x_{j} \operatorname{Cov}\left(r_{i}, r_{j}\right) \\
= & \sum_{k=1}^{n} x_{k}^{2} \frac{1}{24}\left[6\left(b_{k}-a_{k}\right)^{2}+\left(\beta_{k}+\alpha_{k}\right)^{2}+4\left(b_{k}-a_{k}\right)\left(\beta_{k}+\alpha_{k}\right)\right] \\
& +2 \sum_{i>j=1}^{n} x_{i} x_{j} \frac{1}{24}\left[6\left(b_{i}-a_{i}\right)\left(b_{j}-a_{j}\right)+\left(\beta_{i}+\alpha_{i}\right)\left(\beta_{j}+\alpha_{j}\right)+2\left[\left(b_{i}-a_{i}\right)\left(\beta_{j}+\alpha_{j}\right)+\left(b_{j}-a_{j}\right)\left(\beta_{i}+\alpha_{i}\right)\right]\right], \\
& \sum_{k=1}^{n} x_{k}\left[M_{*}\left(r_{k}\right), M^{*}\left(r_{k}\right)\right]+r_{f}\left(1-\sum_{k=1}^{n} x_{k}\right)=\sum_{k=1}^{n} x_{k}\left[a_{k}-\frac{\alpha_{k}}{3}, b_{k}+\frac{\beta_{k}}{3}\right]+r_{f}\left(1-\sum_{k=1}^{n} x_{k}\right) .
\end{aligned}
$$

Zhang [35] proposed the lower possibilistic meanstandard deviation model

$$
\left\{\begin{array}{l}
\min \frac{\sqrt{2}}{6}\left(\sum_{k=1}^{n} \alpha_{k} x_{k}\right) \\
\text { s.t. } \quad \sum_{k=1}^{n}\left(a_{k}-\frac{\alpha_{k}}{3}\right) x_{k}+r_{f}\left(1-\sum_{k=1}^{n} x_{k}\right) \geq \mu, \\
\sum_{k=1}^{n} x_{k} \leq 1, \\
0 \leq d_{k} \leq x_{k} \leq g_{k}, \quad k=1,2, \ldots, n .
\end{array}\right.
$$

The optimal solution to (25) is called a lower possibilistic efficient portfolio. All the lower possibilistic efficient portfolios construct the lower possibilistic efficient frontier.

In this paper, we will assume that the expected return rate of the portfolio is an interval, so as to establish an interval-valued possibilistic mean-variance portfolio model.

From (23) and (24), (22) can be transformed into

$$
\left\{\begin{array}{l}
\min \sum_{k=1}^{n} x_{k}^{2}\left[6\left(b_{k}-a_{k}\right)^{2}+\left(\beta_{k}+\alpha_{k}\right)^{2}+4\left(b_{k}-a_{k}\right)\left(\beta_{k}+\alpha_{k}\right)\right] \\
+2 \sum_{i>j=1}^{n} x_{i} x_{j}\left[6\left(b_{i}-a_{i}\right)\left(b_{j}-a_{j}\right)+\left(\beta_{i}+\alpha_{i}\right)\left(\beta_{j}+\alpha_{j}\right)+2\left[\left(b_{i}-a_{i}\right)\left(\beta_{j}+\alpha_{j}\right)+\left(b_{j}-a_{j}\right)\left(\beta_{i}+\alpha_{i}\right)\right]\right] \\
\text { s.t. } \sum_{k=1}^{n} x_{k}\left[a_{k}-\frac{\alpha_{k}}{3}, b_{k}+\frac{\beta_{k}}{3}\right]+r_{f}\left(1-\sum_{k=1}^{n} x_{k}\right) \geq\left[\mu^{-}, \mu^{+}\right] \\
\sum_{k=1}^{n} x_{k} \leq 1, \\
0 \leq d_{k} \leq x_{k} \leq g_{k}, \quad k=1,2, \ldots, n .
\end{array}\right.
$$


According to Definition 11, (26) can be transformed into

$$
\left\{\begin{array}{l}
\min \sum_{k=1}^{n} x_{k}^{2}\left[6\left(b_{k}-a_{k}\right)^{2}+\left(\beta_{k}+\alpha_{k}\right)^{2}+4\left(b_{k}-a_{k}\right)\left(\beta_{k}+\alpha_{k}\right)\right] \\
+2 \sum_{i>j=1}^{n} x_{i} x_{j}\left[6\left(b_{i}-a_{i}\right)\left(b_{j}-a_{j}\right)+\left(\beta_{i}+\alpha_{i}\right)\left(\beta_{j}+\alpha_{j}\right)+2\left[\left(b_{i}-a_{i}\right)\left(\beta_{j}+\alpha_{j}\right)+\left(b_{j}-a_{j}\right)\left(\beta_{i}+\alpha_{i}\right)\right]\right] \\
\text { s.t. }\left[\sum_{k=1}^{n} x_{k}\left(a_{k}-\frac{\alpha_{k}}{3}\right)+r_{f}\left(1-\sum_{k=1}^{n} x_{k}\right), \sum_{k=1}^{n} x_{k}\left(b_{k}+\frac{\beta_{k}}{3}\right)+r_{f}\left(1-\sum_{k=1}^{n} x_{k}\right)\right] \geq\left[\mu^{-}, \mu^{+}\right] \\
\sum_{k=1}^{n} x_{k} \leq 1, \\
0 \leq d_{k} \leq x_{k} \leq g_{k}, \quad k=1,2, \ldots, n .
\end{array}\right.
$$

According to the order relations $\preccurlyeq_{1}$ and $\preccurlyeq_{2}$ between two interval numbers, we can get

$$
\begin{aligned}
& \int \min \sum_{k=1}^{n} x_{k}^{2}\left[6\left(b_{k}-a_{k}\right)^{2}+\left(\beta_{k}+\alpha_{k}\right)^{2}+4\left(b_{k}-a_{k}\right)\left(\beta_{k}+\alpha_{k}\right)\right] \\
& +2 \sum_{i>j=1}^{n} x_{i} x_{j}\left[6\left(b_{i}-a_{i}\right)\left(b_{j}-a_{j}\right)+\left(\beta_{i}+\alpha_{i}\right)\left(\beta_{j}+\alpha_{j}\right)+2\left[\left(b_{i}-a_{i}\right)\left(\beta_{j}+\alpha_{j}\right)+\left(b_{j}-a_{j}\right)\left(\beta_{i}+\alpha_{i}\right)\right]\right] \\
& \left\{\text { s.t. } \frac{1}{2}\left[\sum_{k=1}^{n} x_{k}\left(a_{k}-\frac{\alpha_{k}}{3}\right)+r_{f}\left(1-\sum_{k=1}^{n} x_{k}\right)+\sum_{k=1}^{n} x_{k}\left(b_{k}+\frac{\beta_{k}}{3}\right)+r_{f}\left(1-\sum_{k=1}^{n} x_{k}\right)\right] \geq \frac{1}{2}\left[\mu^{-}+\mu^{+}\right]\right. \text {, } \\
& \sum_{k=1}^{n} x_{k}\left(a_{k}-\frac{\alpha_{k}}{3}\right)+r_{f}\left(1-\sum_{k=1}^{n} x_{k}\right) \geq \mu^{-} \\
& \sum_{k=1}^{n} x_{k} \leq 1 \\
& 0 \leq d_{k} \leq x_{k} \leq g_{k}, \quad k=1,2, \ldots, n, \\
& \int \min \sum_{k=1}^{n} x_{k}^{2}\left[6\left(b_{k}-a_{k}\right)^{2}+\left(\beta_{k}+\alpha_{k}\right)^{2}+4\left(b_{k}-a_{k}\right)\left(\beta_{k}+\alpha_{k}\right)\right] \\
& +2 \sum_{i>j=1}^{n} x_{i} x_{j}\left[6\left(b_{i}-a_{i}\right)\left(b_{j}-a_{j}\right)+\left(\beta_{i}+\alpha_{i}\right)\left(\beta_{j}+\alpha_{j}\right)+2\left[\left(b_{i}-a_{i}\right)\left(\beta_{j}+\alpha_{j}\right)+\left(b_{j}-a_{j}\right)\left(\beta_{i}+\alpha_{i}\right)\right]\right] \\
& \text { s.t. } \frac{1}{2}\left[\sum_{k=1}^{n} x_{k}\left(a_{k}-\frac{\alpha_{k}}{3}\right)+r_{f}\left(1-\sum_{k=1}^{n} x_{k}\right)+\sum_{k=1}^{n} x_{k}\left(b_{k}+\frac{\beta_{k}}{3}\right)+r_{f}\left(1-\sum_{k=1}^{n} x_{k}\right)\right] \geq \frac{1}{2}\left[\mu^{-}+\mu^{+}\right] \text {, } \\
& \sum_{k=1}^{n} x_{k}\left(b_{k}+\frac{\beta_{k}}{3}\right)+r_{f}\left(1-\sum_{k=1}^{n} x_{k}\right) \geq \mu^{+} \text {, } \\
& \sum_{k=1}^{n} x_{k} \leq 1 \text {, } \\
& 0 \leq d_{k} \leq x_{k} \leq g_{k}, \quad k=1,2, \ldots, n .
\end{aligned}
$$


Then, (28) and (29) are quadratic programming problems that can be solved using Matlab, Lingo, and other software to obtain the possibilistic efficient portfolios.

Definition 12. The optimal solution to (28) or (29) is called an interval-valued possibilistic efficient portfolio.

The lower bounds of all the interval-valued possibilistic efficient portfolios construct the interval-valued lower possibilistic efficient frontier. The upper bounds of all the interval-valued possibilistic efficient portfolios construct the interval-valued upper possibilistic efficient frontier.

\section{Numerical Example}

To illustrate the proposed method, the daily data of six bonds listed on China's Shanghai and Shenzhen bond markets are selected as the original data for analysis. We collect the daily returns of more than 400 trading days from April 2018 to November 2019 as the expected return of bonds. Since the bond price fluctuates, triangular fuzzy numbers are selected as the daily return of the bond. $o_{k t}, c_{k t}, h_{k t}$, and $l_{k t}$ are used to represent the opening, closing, high, and low price of the bond $k$ on the trading day $t$, respectively. $r_{k t}$ is used to represent the return of the bond $k$ on the trading day $t$, set $r_{k t}=\left(a_{k t}, \alpha_{k t}, \beta_{k t}\right)$, where $a_{k t}, \alpha_{k t}, \beta_{k t}$ are calculated as follows:

$$
\begin{aligned}
& a_{k t}=\frac{c_{k t}-o_{k(t-1)}}{o_{k(t-1)}}, \\
& \alpha_{k t}=a_{k t}-L_{k t}, \\
& \beta_{k t}=R_{k t}-a_{k t}, \\
& L_{k t}=\frac{l_{k t}-o_{k(t-1)}}{o_{k(t-1)}}, \\
& R_{k t}=\frac{h_{k t}-o_{k(t-1)}}{o_{k(t-1)}} .
\end{aligned}
$$

Therefore, the expected return of the bond $k$ is

$$
r_{k}=\frac{1}{T_{k}-1} \sum_{t=2}^{T_{k}} r_{k t}
$$

where $T_{k}$ represents the total number of trading days for the bond $k$.

So, formulas (30)-(35) can be used to obtain the triangular fuzzy numbers of the expected returns of the six bonds, as shown in Table 1.

Let the risk-free asset be a Treasury bond. We use the one-year Treasury bond rate as the return rate of the riskless asset. So, we get the return on risk-free asset $r_{f}=2.8 \%$ if the lower bound of investment ratio $x_{k}$ must be $d=\{0.01,0.03,0.01,0.01,0,0.03\}$ and the upper bound $g=\{0.07,0.2,0.3,0.1,0.3,0.35\}$. By solving models (28) and (29), the interval-valued possibilistic efficient portfolios for the different $\bar{\mu} s$ are obtained as shown in Tables 2 and 3.

\begin{tabular}{|c|c|c|c|c|c|c|c|c|}
\hline $\bar{\mu}$ & B1 & B2 & B3 & B4 & B5 & B6 & Risk & $\sum_{k=1}^{6} x_{k}$ \\
\hline $\begin{array}{l}{[0.04,} \\
0.06]\end{array}$ & 0.010 & 0.030 & 0.010 & 0.010 & 0 & 0.229 & 0.018 & 0.289 \\
\hline $\begin{array}{l}{[0.04,} \\
0.08]\end{array}$ & 0.010 & 0.030 & 0.010 & 0.010 & 0 & 0.229 & 0.018 & 0.289 \\
\hline $\begin{array}{l}{[0.04,} \\
0.10]\end{array}$ & 0.010 & 0.030 & 0.010 & 0.010 & 0 & 0.255 & 0.021 & 0.315 \\
\hline $\begin{array}{l}{[0.04,} \\
0.15]\end{array}$ & 0.010 & 0.030 & 0.034 & 0.010 & 0.021 & 0.350 & 0.051 & 0.455 \\
\hline $\begin{array}{l}{[0.04,} \\
0.20]\end{array}$ & 0.010 & 0.030 & 0.087 & 0.010 & 0.062 & 0.350 & 0.106 & 0.549 \\
\hline $\begin{array}{l}{[0.04,} \\
0.30]\end{array}$ & 0.010 & 0.069 & 0.177 & 0.010 & 0.130 & 0.350 & 0.292 & 0.746 \\
\hline $\begin{array}{l}{[0.04,} \\
0.40]\end{array}$ & 0.010 & 0.113 & 0.266 & 0.010 & 0.196 & 0.350 & 0.578 & 0.945 \\
\hline $\begin{array}{l}{[0.04,} \\
0.454]\end{array}$ & 0.010 & 0.106 & 0.300 & 0.010 & 0.300 & 0.274 & 0.822 & 1 \\
\hline $\begin{array}{l}{[0.05,} \\
0.06]\end{array}$ & 0.010 & 0.030 & 0.075 & 0.010 & 0 & 0.350 & 0.060 & 0.475 \\
\hline $\begin{array}{l}{[0.05,} \\
0.08]\end{array}$ & 0.010 & 0.030 & 0.075 & 0.010 & 0 & 0.350 & 0.060 & 0.475 \\
\hline $\begin{array}{l}{[0.05,} \\
0.10]\end{array}$ & 0.010 & 0.030 & 0.075 & 0.010 & 0 & 0.350 & 0.060 & 0.475 \\
\hline $\begin{array}{l}{[0.05,} \\
0.15]\end{array}$ & 0.010 & 0.030 & 0.065 & 0.010 & 0.012 & 0.350 & 0.061 & 0.477 \\
\hline $\begin{array}{l}{[0.05,} \\
0.20]\end{array}$ & 0.010 & 0.030 & 0.097 & 0.010 & 0.069 & 0.350 & 0.120 & 0.566 \\
\hline $\begin{array}{l}{[0.05,} \\
0.30]\end{array}$ & 0.010 & 0.074 & 0.186 & 0.010 & 0.136 & 0.350 & 0.316 & 0.766 \\
\hline $\begin{array}{l}{[0.05,} \\
0.40]\end{array}$ & 0.010 & 0.118 & 0.275 & 0.010 & 0.203 & 0.350 & 0.612 & 0.966 \\
\hline $\begin{array}{l}{[0.05,} \\
0.454]\end{array}$ & 0.010 & 0.199 & 0.300 & 0.010 & 0.300 & 0.181 & 0.917 & 1 \\
\hline
\end{tabular}

TABLE 1: Parameter values of the triangular fuzzy number of the six bond returns.

\begin{tabular}{lccc}
\hline Code & $a_{k}$ & $\alpha_{k}$ & $\beta_{k}$ \\
\hline B1 & -0.018 & 0.421 & 0.500 \\
B2 & 0.237 & 0.646 & 0.524 \\
B3 & 0.295 & 0.694 & 0.632 \\
B4 & 0.021 & 0.260 & 0.254 \\
B5 & 0.302 & 0.732 & 0.810 \\
B6 & 0.197 & 0.315 & 0.107 \\
\hline
\end{tabular}

TABLE 2: The investment proportion and risk for different $\bar{\mu} s$ in (28).

Figures 1 and 2 give some interval-valued possibilistic efficient portfolios for models (28) and (29), respectively.

From Table 2, we can find the following.

(1) When the lower limit of the minimum threshold of the expected rate of return $\mu^{-}$remains unchanged, the investment proportion of B6 will increase first as the upper limit $\mu^{+}$increases. When the investment proportion of $\mathrm{B} 6$ reaches its ceiling at 0.350 , investors will increase the investment proportion of $\mathrm{B} 3$ and $\mathrm{B} 5$, which in turn is the investment proportion of B2. As $\mu^{+}$continues to increase, investors will reduce the investment proportion of $\mathrm{B} 6$. Meanwhile, 
TABLE 3: The investment proportion and risk for different $\bar{\mu} s$ in (29).

\begin{tabular}{lcccccccc}
\hline $\bar{\mu}$ & $\mathrm{B} 1$ & $\mathrm{~B} 2$ & $\mathrm{~B} 3$ & $\mathrm{~B} 4$ & $\mathrm{~B} 5$ & B6 & Risk & $\sum_{k=1}^{6} x_{k}$ \\
\hline$[0.04,0.06]$ & 0.010 & 0.030 & 0.010 & 0.010 & 0 & 0.106 & 0.008 \\
{$[0.04,0.08]$} & 0.010 & 0.030 & 0.010 & 0.010 & 0 & 0.181 & 0.013 \\
{$[0.04,0.10]$} & 0.010 & 0.030 & 0.016 & 0.010 & 0.011 & 0.221 & 0.022 \\
{$[0.04,0.15]$} & 0.010 & 0.030 & 0.042 & 0.010 & 0.031 & 0.350 & 0.060 \\
{$[0.04,0.20]$} & 0.010 & 0.054 & 0.087 & 0.010 & 0.067 & 0.350 & 0.123 \\
{$[0.04,0.30]$} & 0.010 & 0.118 & 0.170 & 0.010 & 0.133 & 0.350 & 0.334 \\
{$[0.04,0.40]$} & 0.010 & 0.168 & 0.255 & 0.010 & 0.207 & 0.350 & 0.655 \\
{$[0.05,0.06]$} & 0.010 & 0.030 & 0.010 & 0.010 & 0 & 0.143 & 0.010 \\
{$[0.05,0.08]$} & 0.010 & 0.030 & 0.010 & 0.010 & 0 & 0.218 & 0.017 \\
{$[0.05,0.10]$} & 0.010 & 0.030 & 0.010 & 0.010 & 0 & 0.293 & 0.026 \\
{$[0.05,0.15]$} & 0.010 & 0.030 & 0.045 & 0.010 & 0.029 & 0.350 & 0.060 \\
{$[0.05,0.20]$} & 0.010 & 0.054 & 0.087 & 0.010 & 0.067 & 0.350 & 0.273 \\
{$[0.05,0.30]$} & 0.010 & 0.118 & 0.170 & 0.010 & 0.133 & 0.350 & 0.353 \\
{$[0.05,0.40]$} & 0.010 & 0.168 & 0.255 & 0.010 & 0.207 & 0.350 & 0.334 \\
\hline
\end{tabular}

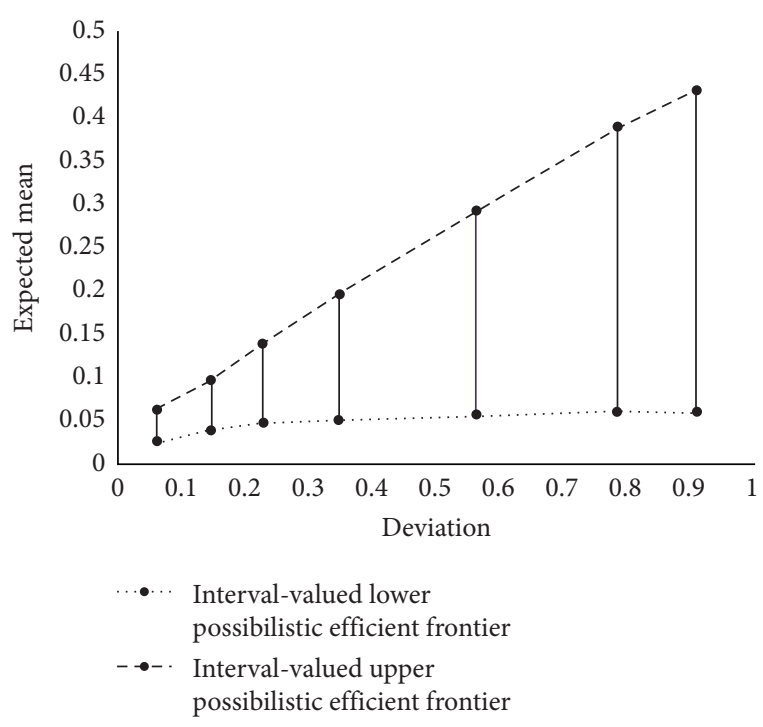

FIGURE 1: Some interval-valued possibilistic efficient portfolios for model (28).

the investment proportions of B3 and B5 will be increased to their respective investment caps. The investment proportion of B2 also increases along with the increase of $\mu^{+}$. Finally, the total investment proportion of the six risky assets is $100 \%$. At this point, the risk-free asset investment ratio is 0 . During the investment process, the investment ratios of $\mathrm{B} 1$ and $\mathrm{B} 4$ are kept at their respective minimum investment ratios.

(2) When the lower limit of the minimum expected rate of return $\mu^{-}$is unchanged, the investment risk will increase alongside the increase of the upper limit $\mu^{+}$, which just confirms that a high return is accompanied by a high risk, and conversely, that a high risk requires a corresponding high return as compensation.

(3) By relation $\preccurlyeq_{1}$ know that, for the same $\mu^{+}$, with the increase of $\mu^{-}$, the increase in the risk of a portfolio and the investment proportion of risky assets.

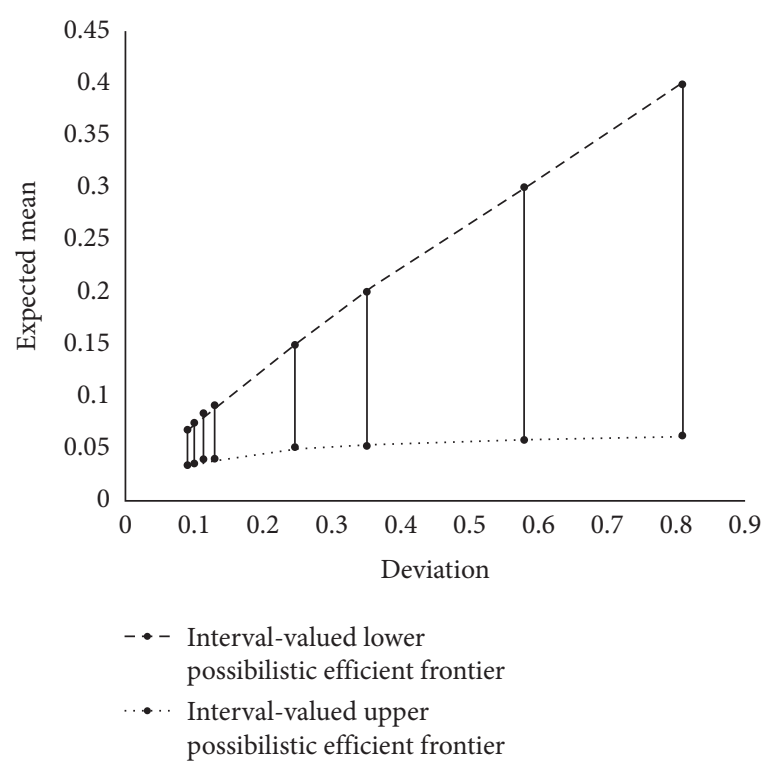

FIGURE 2: Some interval-valued possibilistic efficient portfolios for model (29).

(4) According to (14) and the historical rate of return of various assets in the portfolio, when $\mu^{-}$or $\mu^{+}$increases to a certain extent, there will be no feasible solution for model (28).

For model (29), the law shown in Table 3 is similar to that in Table 2, but it differs slightly as follows.

When $\mu^{-}$is unchanged, with the increase of $\mu^{+}$, investors first increase the investment proportion of B6. Different from model (28), in (29), before the investment proportion of $\mathrm{B} 6$ reaches its investment limit of 0.350 , the investment proportions of B5 and B3 start to increase.

Besides, by comparing Tables 2 and 3, we can find that for the same $\bar{\mu}$, model (29) invests less in risky assets and takes less risk. In other words, we allocate assets according to the proportion of investment obtained by model (29) and can obtain the same return as model (28) by taking less risk.

Figures 1 and 2 show the relationship between the interval-valued expected return rate and risk. For example, in 
Figure 1 , when $\bar{\mu}=[0.04,0.10]$, the interval of expected return rate is $[0.042,0.098]$, and the risk of the portfolio is 0.1449 ; that is, the risk assumed by the investor is 0.1449 .

From Figures 1 and 2, we can see the following:

(1) As the expected return rate of the portfolio increases, the risk also increases correspondingly.

(2) When the lower limit of the interval of expected return rate remains unchanged, the risk of the portfolio will increase with the increase of the upper limit. By contrast, when the upper limit of the interval of expected return rate remains unchanged, the risk of the portfolio will increase with the increase of the lower limit.

\section{Model Discussion}

In this paper, the interval-valued possibilistic mean (see Definition 6) is used to measure the expected return rate of the portfolio. The portfolio selection model (21) is converted into an interval-valued portfolio model (22), and Definition 11 is used to convert model (22) into the following:

$$
\begin{cases}\min & \operatorname{Var}(R)=\sum_{k=1}^{n} x_{k}^{2} \operatorname{Var}\left(r_{k}\right)+2 \sum_{i>j=1}^{n} x_{i} x_{j} \operatorname{Cov}\left(r_{i}, r_{j}\right) \\ \text { s.t. } & \sum_{k=1}^{n} \frac{M_{*}\left(r_{k}\right)+M^{*}\left(r_{k}\right)}{2} x_{k}+r_{f}\left(1-\sum_{k=1}^{n} x_{k}\right) \geq \frac{\mu^{-}+\mu^{+}}{2}, \\ M_{*}\left(r_{k}\right)+r_{f}\left(1-\sum_{\mathrm{k}=1}^{n} x_{k}\right) \geq \mu^{-}, \\ \sum_{k=1}^{n} x_{k} \leq 1, \\ 0 \leq d_{k} \leq x_{k} \leq g_{k}, \quad k=1,2, \ldots, n,\end{cases}
$$

$$
\begin{cases}\min & \operatorname{Var}(R)=\sum_{k=1}^{n} x_{k}^{2} \operatorname{Var}\left(r_{k}\right)+2 \sum_{i>j=1}^{n} x_{i} x_{j} \operatorname{Cov}\left(r_{i}, r_{j}\right) \\ \text { s.t. } & \sum_{k=1}^{n} \frac{M_{*}\left(r_{k}\right)+M^{*}\left(r_{k}\right)}{2} x_{k}+r_{f}\left(1-\sum_{k=1}^{n} x_{k}\right) \geq \frac{\mu^{-}+\mu^{+}}{2}, \\ M^{*}\left(r_{k}\right)+r_{f}\left(1-\sum_{\mathrm{k}=1}^{n} x_{k}\right) \geq \mu^{+}, \\ \sum_{k=1}^{n} x_{k} \leq 1, \\ 0 \leq d_{k} \leq x_{k} \leq g_{k}, \quad k=1,2, \ldots, n .\end{cases}
$$

If we use the crisp possibilistic mean value (see Definition 7) when measuring the expected return rate of a portfolio, the portfolio selection model (21) is transformed into

$$
\left\{\begin{array}{l}
\min \quad \operatorname{Var}(R)=\sum_{k=1}^{n} x_{k}^{2} \operatorname{Var}\left(r_{k}\right)+2 \sum_{i>j=1}^{n} x_{i} x_{j} \operatorname{Cov}\left(r_{i}, r_{j}\right) \\
\text { s.t. } \quad \sum_{k=1}^{n} \frac{M_{*}\left(r_{k}\right)+M^{*}\left(r_{k}\right)}{2} x_{k}+r_{f}\left(1-\sum_{k=1}^{n} x_{k}\right) \geq \mu, \\
\sum_{k=1}^{n} x_{k} \leq 1, \\
0 \leq d_{k} \leq x_{k} \leq g_{k}, \quad k=1,2, \ldots, n,
\end{array}\right.
$$

where $\mu^{-}=\mu^{+}=\mu$. This is the possibilistic mean-variance model proposed by Zhang and Wang [34].

From (36)-(38), we can find that (36) and (37) have more constraints. And while the optimal solution to (36) or (37) must be a feasible solution to (38), it does not necessarily have to be the reverse. In addition, compared with (38), models (36) and (37) are more flexible in describing the minimum expected return expected by investors.

In Table 4, the numerical example in Section 4 is solved by using model (38), and the optimal investment ratio is obtained.

As can be seen in Table 4, with the increase of $\mu$, the investment proportion of B6 will increase first, followed by $\mathrm{B} 5, \mathrm{~B} 3$, and $\mathrm{B} 2$; and finally, the investment proportion of risk assets will reach $100 \%$. At this time, the investment proportion of risk-free assets will be 0 . With the increase of $\mu$, the investment risk also increases, which is consistent with the high return accompanied by high risk.

At the same time, by comparing models (36)-(38), it can be found that the optimal solutions of (36) and (37) must be the feasible solution of (38). Therefore, the optimal solution of (38) should not be greater than the optimal solutions of (36) and (37). Table 5 shows the risk comparison of different expected return thresholds in models (36) and (38). Some interval-valued possibilistic efficient portfolios of model (36) as well as the lower possibilistic effective portfolios proposed by Zhang [35] are shown in Figure 3. Figure 4 shows some interval-valued possibilistic efficient portfolios of model (36) as well as the possibilistic effective portfolios of model (38).

When $\bar{\mu}=[0.04,0.06], \quad(1 / 2)\left(\mu^{-}+\mu^{+}\right)=0.05$. So, $(1 / 2)\left(\mu^{-}+\mu^{+}\right)=\mu$. By comparing the value of the objective function in model (36), the minimum risk borne by investors is obviously greater than the minimum risk borne by investors determined by model (38). This rule still holds for other values of $\bar{\mu}$, which just verifies that the optimal solution of model (36) mentioned above is the feasible solution of model (38). Similarly, the objective function values of models (37) and (38) have the same relationship.

As shown in Figures 3 and 4, the (lower) possibilistic efficient frontier lies exactly between the interval-valued lower and upper possibilistic efficient frontiers. Since the expected return rate of assets in our model is not a fixed number but an interval, the expected return rate of the portfolio is also an interval. Therefore, this model is 
TABle 4: The investment proportion and risk for different $\mu$ s in (38).

\begin{tabular}{lcccccccc}
\hline$\mu$ & B1 & B2 & B3 & B4 & B5 & B6 & Risk & $\sum_{k=1}^{6} x_{k}$ \\
\hline 0.050 & 0.010 & 0.030 & 0.010 & 0.010 & 0 & 0.106 & 0.008 & 0.166 \\
0.055 & 0.010 & 0.030 & 0.010 & 0.010 & 0 & 0.143 & 0.010 & 0.203 \\
0.060 & 0.010 & 0.030 & 0.010 & 0.010 & 0 & 0.181 & 0.013 & 0.241 \\
0.065 & 0.010 & 0.030 & 0.010 & 0.010 & 0 & 0.218 & 0.017 & 0.278 \\
0.070 & 0.010 & 0.030 & 0.010 & 0.010 & 0 & 0.255 & 0.021 & 0.315 \\
0.075 & 0.010 & 0.030 & 0.010 & 0.010 & 0 & 0.293 & 0.026 & 0.353 \\
0.083 & 0.010 & 0.030 & 0.010 & 0.010 & 0.001 & 0.350 & 0.034 & 0.411 \\
0.095 & 0.010 & 0.030 & 0.034 & 0.010 & 0.021 & 0.350 & 0.051 & 0.455 \\
0.100 & 0.010 & 0.030 & 0.045 & 0.010 & 0.029 & 0.350 & 0.060 & 0.474 \\
0.120 & 0.010 & 0.030 & 0.087 & 0.010 & 0.062 & 0.350 & 0.106 & 0.549 \\
0.125 & 0.010 & 0.030 & 0.097 & 0.010 & 0.070 & 0.350 & 0.120 & 0.567 \\
0.170 & 0.010 & 0.069 & 0.177 & 0.010 & 0.130 & 0.350 & 0.292 & 0.746 \\
0.175 & 0.010 & 0.074 & 0.186 & 0.010 & 0.136 & 0.350 & 0.316 & 0.766 \\
0.220 & 0.010 & 0.113 & 0.266 & 0.010 & 0.196 & 0.350 & 0.578 & 0.945 \\
0.225 & 0.010 & 0.118 & 0.275 & 0.010 & 0.203 & 0.350 & 0.612 & 0.966 \\
0.085 & 0.010 & 0.126 & 0.291 & 0.010 & 0.213 & 0.350 & 0.684 & 1 \\
\hline
\end{tabular}

TABLE 5: The risk comparison between model (36) and model (38) for different $\mu$ s and $\bar{\mu}$.

\begin{tabular}{lccc}
\hline$\mu$ & Risk in (38) & $\bar{\mu}$ & Risk in (36) \\
\hline 0.050 & 0.004 & {$[0.04,0.06]$} & 0.018 \\
0.065 & 0.004 & {$[0.05,0.08]$} & 0.060 \\
0.070 & 0.004 & {$[0.04,0.10]$} & 0.021 \\
0.095 & 0.006 & {$[0.04,0.15]$} & 0.051 \\
0.100 & 0.008 & {$[0.05,0.15]$} & 0.061 \\
0.120 & 0.013 & {$[0.04,0.20]$} & 0.106 \\
0.125 & 0.015 & {$[0.05,0.20]$} & 0.120 \\
0.170 & 0.037 & {$[0.04,0.30]$} & 0.292 \\
0.175 & 0.040 & {$[0.05,0.30]$} & 0.316 \\
0.220 & 0.081 & {$[0.04,0.40]$} & 0.578 \\
0.225 & 0.087 & {$[0.05,0.40]$} & 0.612 \\
\hline
\end{tabular}

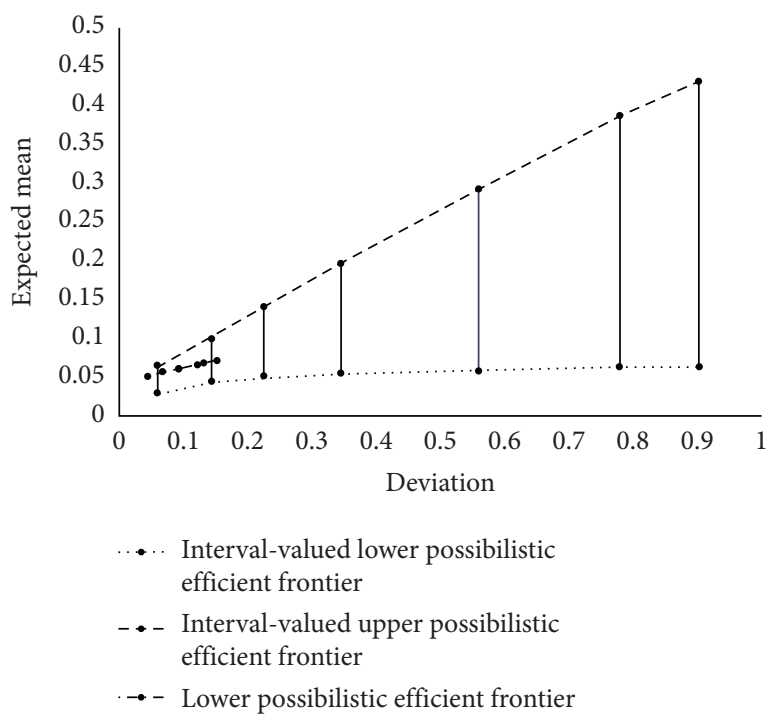

FIGURE 3: Some interval-valued possibilistic efficient portfolios and lower possibilistic efficient portfolios.

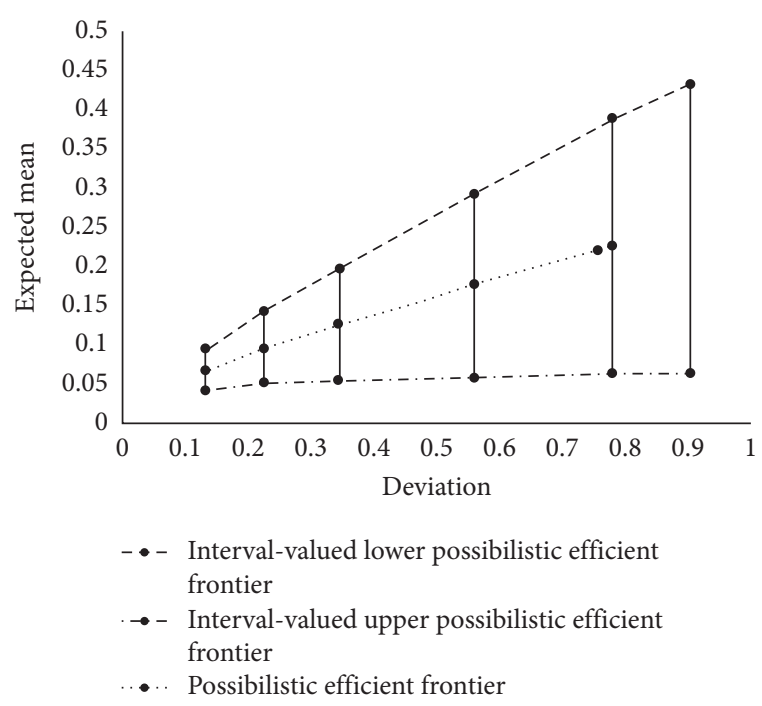

FIGURE 4: Some interval-valued possibilistic efficient portfolios and possibilistic efficient portfolios.

applicable to a wider range of application and conforms to the investment behaviour and psychology of investors in the financial market.

\section{Conclusions}

In this paper, we treat the return rates of assets as fuzzy numbers and measure the expected return rates using the interval-valued possibilistic mean of fuzzy numbers. Thus, an interval-valued possibilistic mean-variance portfolio selection model is constructed. In this model, the expected return rate and the lower bound of the expected return rate of the portfolio are regarded as intervals using possibility theory. Doing so, one can more accurately measure investors' expected returns. In the real process of making decisions, an investor's expected return rate on a portfolio is often not a fixed value but spans a certain range of numbers. Therefore, the expected return rate and the lower bound of the expected return rate are regarded as more realistic intervals. An application of the portfolio diversification problem is provided where the available assets include six bonds. The results show that the lower limit of the expected return rate is more flexible in this method. Through comparison with the models proposed by Zhang and Wang [34] and Zhang [35], it can be found that this model can be more widely applied and can more flexibly describe the expected return rate of an investment portfolio.

\section{Data Availability}

The data used to support the findings of this study are available from the corresponding author upon request.

\section{Conflicts of Interest}

The authors declare that they have no conflicts of interest. 


\section{References}

[1] H. Markowitz, "Portfolio selection," The Journal of Finance, vol. 7, no. 1, pp. 77-91, 1952.

[2] W. F. Sharpe, Portfolio Theory and Capital Markets, McGrawHill, New York, NY, USA, 1970.

[3] R. C. Merton, "An analytic derivation of the efficient portfolio frontier," The Journal of Financial and Quantitative Analysis, vol. 7, no. 4, pp. 1851-1872, 1972.

[4] J.-S. Pang, "A new and efficient algorithm for a class of portfolio selection problems," Operations Research, vol. 28, no. 3, pp. 754-767, 1980.

[5] A. F. Perold, "Large-scale portfolio optimization," Management Science, vol. 30, no. 10, pp. 1143-1160, 1984.

[6] H. Markowitz, Portfolio Selection: Efficient Diversification of Investments, Yale University Press, New York, NY, USA, 1959.

[7] H. Konno, H. Shirakawa, and H. Yamazaki, "A mean-absolute deviation-skewness portfolio optimization model," Annals of Operations Research, vol. 45, no. 1, pp. 205-220, 1993.

[8] M. G. Speranza, "Linear programming model for portfolio optimization," Finance, vol. 14, no. 1, pp. 107-123, 1993.

[9] S. Basak and A. Shapiro, "Value-at-risk-based risk management: optimal policies and asset prices," Review of Financial Studies, vol. 14, no. 2, pp. 371-405, 2001.

[10] F.-Y. Chen, "Analytical VaR for international portfolios with common jumps," Computers \& Mathematics with Applications, vol. 62, no. 8, pp. 3066-3076, 2011.

[11] S. X. Chen and C. Y. Tang, "Nonparametric inference of valueat-risk for dependent financial returns," Journal of Financial Econometrics, vol. 3, no. 2, pp. 227-255, 2005.

[12] Y. Wei, Y. P. Wang, and H. J. Xuan, "Fuzzy multi-objective portfolio model based on semi-variance-semi-absolute deviation risk measures," Soft Computing-A Fusion of Foundations, Methodologies \& Applications, vol. 23, pp. 8159-8179, 2019.

[13] B. Liu, "Why is there a need for uncertainty theory?," Journal of Uncertain Systems, vol. 6, no. 1, pp. 3-10, 2012.

[14] Q. Zhang, X. Huang, and C. Zhang, "A mean-risk index model for uncertain capital budgeting," Journal of the Operational Research Society, vol. 67, no. 5, pp. 761-770, 2014.

[15] L. A. Zadeh, "Fuzzy sets," Information and Control, vol. 8, no. 3, pp. 338-353, 1965.

[16] J. Watada, "Fuzzy portfolio selection and its applications to decision making," Tatra Mountains Mathematical Publication, vol. 13, pp. 219-248, 1997.

[17] S. Ramaswamy, "Portfolio selection using fuzzy decision theory," Working Paper of the Bank for International Settlements, 1998.

[18] T. León, V. Liern, and E. Vercher, "Viability of infeasible portfolio selection problems: a fuzzy approach," European Journal of Operational Research, vol. 139, no. 1, pp. 178-189, 2002.

[19] S. Wang and S. Zhu, "On fuzzy portfolio selection problems," Fuzzy Optimization and Decision Making, vol. 1, no. 4, pp. 361-377, 2002.

[20] S.-h. Lee, "A fuzzy multi-objective programming approach for determination of resilient supply portfolio under supply failure risks," Journal of Purchasing and Supply Management, vol. 23, no. 3, pp. 211-220, 2017.

[21] Y. Y. Sui, J. S. Hu, and F. Ma, "A possibilistic portfolio model with fuzzy liquidity constraint," Complexity, vol. 2020, no. 3, p. 10, 2020.

[22] R.-C. Tsaur, "Fuzzy portfolio model with fuzzy-input return rates and fuzzy-output proportions," International Journal of Systems Science, vol. 46, no. 3, pp. 438-450, 2013.
[23] J. Zhou, X. Li, and W. Pedrycz, "Mean-semi-entropy models of fuzzy portfolio selection," IEEE Transactions on Fuzzy Systems, vol. 24, no. 6, pp. 1627-1636, 2016.

[24] X. Zhang, W.-G. Zhang, and R. Cai, "Portfolio adjusting optimization under credibility measures," Journal of Computational and Applied Mathematics, vol. 234, no. 5, pp. 1458-1465, 2010.

[25] L. A. Zadeh, "Fuzzy sets as a basis for a theory of possibility," Fuzzy Sets and Systems, vol. 1, no. 1, pp. 3-28, 1978.

[26] D. Dubois and H. Prade, "The mean value of a fuzzy number," Fuzzy Sets and Systems, vol. 24, no. 3, pp. 279-300, 1978.

[27] C. Carlsson and R. Fullér, Possibility for Decision: A Possibilistic Approach to Real Life Decisions, Springer, Berlin, Germany, 2011.

[28] I. Georgescu, Possibility Theory and the Risk, Springer, Berlin, Germany, 2012.

[29] H. Tanaka, H. Nakayama, and A. Yanagimoto, "Possibility portfolio selection," Proceedings of the 1995 IEEE International Conference on Fuzzy Systems, pp. 77-91, Yokohama, Japan, March 1995.

[30] C. Carlsson and R. Fullér, "On possibilistic mean value and variance of fuzzy numbers," Fuzzy Sets and Systems, vol. 122, no. 2, pp. 315-326, 2001.

[31] C. Carlsson, R. Fullér, and P. Majlender, "A possibilistic approach to selecting portfolios with highest utility score," Fuzzy Sets and Systems, vol. 131, no. 1, pp. 13-21, 2002.

[32] W. G. Zhang and Z. K. Nie, "On possibilistic variance of fuzzy numbers," Rough Sets, Fuzzy Sets, Data Mining, and Granular Computing, vol. 2639, pp. 398-402, 2003.

[33] R. Fullér and P. Majlender, "On weighted possibilistic mean and variance of fuzzy numbers," Fuzzy Sets and Systems, vol. 136, no. 3, pp. 363-374, 2003.

[34] W. G. Zhang and Y. L. Wang, "Using fuzzy possibilistic mean and variance in portfolio selection model," Computational Intelligence and Security, vol. 3801, pp. 291-296, 2003.

[35] W.-G. Zhang, "Possibilistic mean-standard deviation models to portfolio selection for bounded assets," Applied Mathematics and Computation, vol. 189, no. 2, pp. 1614-1623, 2007.

[36] W.-G. Zhang, Y.-L. Wang, Z.-P. Chen, and Z.-K. Nie, "Possibilistic mean-variance models and efficient frontiers for portfolio selection problem," Information Sciences, vol. 177, no. 13, pp. 2787-2801, 2007.

[37] W.-G. Zhang, W.-L. Xiao, and Y.-L. Wang, "A fuzzy portfolio selection method based on possibilistic mean and variance," Soft Computing, vol. 13, no. 6, pp. 627-633, 2009.

[38] S.-T. Liu and R.-T. Wang, "A numerical solution method to interval quadratic programming," Applied Mathematics and Computation, vol. 189, no. 2, pp. 1274-1281, 2007.

[39] V. Mohagheghi, S. M. Mousavi, and B. Vahdani, "A new optimization model for project portfolio selection under interval-valued fuzzy environment," Arabian Journal for Science and Engineering, vol. 40, no. 11, pp. 3351-3361, 2015.

[40] T. M. Mohaghegh, M. Keyanpour, and W. A. Lodwick, "Solving interval linear programming problems with equality constraints using extended interval enclosure solutions," Soft Computing, vol. 23, pp. 7439-7449, 2019.

[41] E. Garajová, M. Hladík, and M. Rada, "Interval linear programming under transformations: optimal solutions and optimal value range," Central European Journal of Operations Research, vol. 27, no. 3, pp. 601-614, 2019.

[42] S. C. Tong, "Interval number and fuzzy number linear programmings," Fuzzy Sets and Systems, vol. 66, no. 3, pp. 301-306, 1994. 
[43] K. K. Lai, S. Y. Wang, J. P. Xu, S. S. Zhu, and Y. Fang, "A class of linear interval programming problems and its application to portfolio selection," IEEE Transactions on Fuzzy Systems, vol. 10, no. 6, pp. 698-704, 2002.

[44] H. Ishibuchi and H. Tanaka, "Formulation and analysis of linear programming problem with interval coefficients," Japan Industrial Management Association, vol. 40, pp. 320-329, 1989.

[45] H. Ishibuchi and H. Tanaka, "Multiobjective programming in optimization of the interval objective function," European Journal of Operational Research, vol. 48, no. 2, pp. 219-225, 1990.

[46] D. Dubois and H. Prade, Fuzzy Sets and Systems: Theory and Applications, Academic Press, New York, NY, USA, 1980.

[47] E. Hansen, Global Optimization Using Interval Analysis, Marcel Dekker, New York, NY, USA, 1992.

[48] G. Alefeld and J. Herzberger, Introduction to Interval Computations, Academic Press, New York, NY, USA, 1983. 